\title{
Trapping Neutral Atoms with Electromagnetic Fields
}

\author{
J. Dalibard and C. Salomon, Paris
}

(Laboratoire de Spectroscopie Hertzienne de l'Ecole Normale Supérieure)

\begin{abstract}
Neutral atoms have now been trapped in inhomogeneous magnetic fields or laser fields. Densities up to $10^{12}$ atoms $/ \mathrm{cm}^{3}$ have been obtained, with storage times of several minutes. The very low temperature of these trapped atoms (below one millikelvin) opens the way to new physical phenomena.
\end{abstract}

Controlling the motion of charged particles by electromagnetic fields is now a well mastered technique. Its applications range from particle accelerators to the trapping of a single ion, and include the familiar cathode ray tube. The situation is much different for neutral species, as the means of influencing them are so sparse. For example, until 1985, the only way to confine a neutral atom was in a cell. However, during these last three years, several atomic traps have been demonstrated, based either on the action of an inhomogeneous static magnetic field (magnetic trap) or of a laser field (light trap). Here, we briefly review these two kinds of trap, with the aim of giving an overview of the potentialities offered by these new devices.

Neutral atom traps should have applications in various branches of physics. Spectroscopists and atomic clock physicists may find in the very slow atoms emerging from a trap a way to improve the precision of their measurements. Moreover these traps could lead to the observation of collective quantum effects such as Bose Einstein condensation for spin polarized hydrogen. One avoids in these traps the problem of molecular recombination of atomic hydrogen on the walls of the cell.

A general characteristic of neutral atom traps is their very shallow depth, which is inherent in the physical principle involved in the confinement: to confine atoms near a given point, the atomic energy levels are curved so that the energy of the atomic level occupied by the atom is minimal at the centre of the trap. The inhomogeneous shift of the energy level is produced either by a magnetic field (Zeeman shift) or by a laser field (light shift). In the first case, the energy depth of the trap $U_{M}$ is given by $U_{\mathrm{M}} \cong \mu_{\mathrm{B}} \Delta B$ where $\mu_{\mathrm{B}}$ is the Bohr magneton, and $\triangle B$ the difference between the maximal and minimal values of the magnetic field. For a typical $\Delta B$ of $1 \mathrm{~T}$, one gets a depth $U_{M} \cong 0.7 \mathrm{~K}$. For a laser field, the light shift of the energy levels involved in a transition, quasi-resonant with the laser, can be estimated to be $U_{\mathrm{L}} \cong d E_{\mathrm{L}}$, where $d$ is the electric dipole moment of the transition, and $E_{\mathrm{L}}$ the laser electric field. A CW laser of $1 \mathrm{~W}$ power, focussed on $10 \mu \mathrm{m}$, produces in sodium atoms a depth $U_{\mathrm{L}} \cong 2 \mathrm{~K}$.

Because of these very small depths, the filling and the stabilization of the traps are quite difficult. One needs very slow atoms, whose temperature is smaller than the depth of the trap. Such slow atoms can either be produced by the usual cryogenic techniques, or by the recently-developed laser deceleration and cooling techniques [1-3]. Once trapped, the atoms can be kept in the trap only if the various heating mechanisms - collisions with the background gas, heating inherent to laser trapping techniques - can be overcome. One thus needs a very good vacuum (pressure $<10^{-9}$ Torr) and possibly an additional cooling mechanism.

\section{Magnetic Traps}

The magnetic trap was the first device to be used for confining neutral atomic size particles. In 1977, K. Kügler, W. Paul and $U$. Trinks kept neutrons in a toroidal superconducting magnet ring for $20 \mathrm{mi}-$ nutes [4]. In this experiment, realized at the ILL Grenoble, the azimuthal velocity of the neutrons in the storage ring was in the range $7-20 \mathrm{~m} / \mathrm{s}$, and the transverse velocity was lower than $4 \mathrm{~m} / \mathrm{s}$. Neutrons were injected along a radius of the ring, and were deflected in the ring by a totally reflecting mirror system. This mirror system was then promptly taken out of the ring before the neutrons had completed their first turn.

A naïve observer would have thought that a magnetic trap for neutral atoms would have been realized immediately after this. The magnetic moment of an atom is indeed two to three orders of magnitude larger than the magnetic moment of a neutron, at least if the atom has a non zero electronic angular momentum in its ground state. The magnetic force in a given magnetic field is then increased by the same factor. However, eight more years were to pass before the first successful magnetic trapping of neutral atoms.

The problems are indeed very different for atoms and neutrons. First, one needs to find a source of slow atoms. Up to recent times, this was not easy as in a normal thermal atomic beam, there is a deficiency of slow atoms with respect to the Maxwell-Boltzmann distribution. Laser deceleration of atomic beams (see Fig. 1) has thus been a crucial step to the production of trappable slow atoms. Second, one has to find a way of introducing the atoms into the magnetic trap, equivalent to the removal of the mirror system used for neutrons. Here also, laser cooling constitutes an elegant way of extracting a sufficient amount of kinetic atomic energy.

The first magnetic trap for neutral atoms (sodium) was achieved by $W$. Phillips, $\mathrm{H}$. Metcalf and co-workers at NBS Washington [5]. The quadrupole trap (initially proposed by W. Paul) is made of two parallel coils with current in opposite directions. Atoms with a positive magnetic quantum number along the axis, defined by the local magnetic field ("Iow field seekers"), are then attracted towards the centre of the trap, where the field is zero. The maximum field in the trap is 250 Gauss leading to a maximum trappable velocity of $3.5 \mathrm{~m} / \mathrm{s}$. The authors have checked that for such slow atoms, the magnetic moment follows the magnetic field adiabatically: the probability for non adiabatic or Majorana transitions, which would reverse the magnetic moment, is very small.

To fill the trap, the current in the coils is first set to zero and atoms are decelera- 


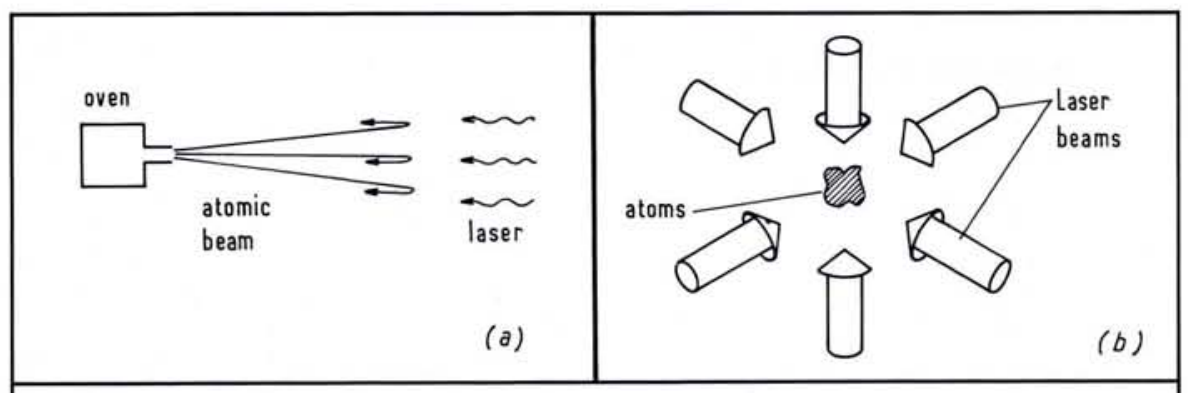

Fig. 1 - Control of atomic motion using the resonant laser "scattering force". The average scattering force exerted by a plane running laser wave on an atom can be written:

$$
\vec{f}=n \hbar \overrightarrow{k_{L}}
$$

where $\vec{k}_{\perp}$ is the wave vector of the light, parallel to the Poynting vector, and $n$ is the number of fluorescence cycles per unit time. Indeed, in such cycles, the atom absorbs laser photons (momentum $\bar{h} \vec{k}_{\mathrm{L}}$ ) and emits spontaneous fluorescence photons in random directions (average momentum $\overrightarrow{0}$ ). This scattering force can be used for:

(a) Deceleration of an atomic beam: in a distance of the order of $1 \mathrm{~m}$, it is possible to stop an atomic beam (sodium, rubidium, caesium, etc.) using a resonant laser beam. An important problem in this slowing down process is to keep the resonance condition between the atomic frequency $\omega_{A}$ and the Doppler shifted laser frequency $\omega_{L}-$ $\vec{k}_{\mathrm{L}} \cdot \vec{v}$. Two solutions have proved to be efficient: spatial change of the atomic frequency using an inhomogeneous magnetic field, and temporal change of the laser frequency (for a review, see [1]).

(b) 3-dimensional cooling of a dilute gas or "optical molasses". Atoms are irradiated by three pairs of opposed laser running waves, detuned red from resonance $\left(\omega_{\mathrm{L}}<\right.$ $\left.\omega_{A}\right)$. Because of the Doppler effect a moving atom will interact more with the waves which are counter-propagating to its velocity lapparent frequency $\omega_{\mathrm{L}}-\vec{k}_{\mathrm{L}} \cdot \vec{v}$ larger than $\omega_{L}$, and close to $\left.\omega_{A}\right)$ than with the waves which are copropagating $\left(\omega_{L}-k \cdot v<\right.$ $\left.\omega_{\mathrm{L}}<\omega_{\mathrm{A}}\right)$. Therefore, the atom experiences a force opposed to its velocity: the atom's velocity gets very quickly damped. This cooling scheme works as long as the six waves have a low enough intensity so that it is possible to add independently their effect. A steady state is obtained when this radiative cooling is counter-balanced by the heating due to the random character of the spontaneous emission processes. This steady state is reached in a typical time $\tau$ given by $m / \hbar k_{L}^{2}$ where $m$ is the atomic mass. For sodium atoms illuminated on their $D_{2}$ resonance line, $\tau$ is about $10 \mu \mathrm{s}$ so that the photon bath can be thought of as a very viscous medium called "optical molasses". The equilibrium velocity distribution can be shown to be close to a Maxwell-Boltzmann distribution with an effective temperature given by $k_{\mathrm{B}} T=\hbar \Gamma / 2$ where $\Gamma^{-1}$ is the radiative lifetime of the excited state of the transition (for sodium atoms, $T=240 \mu \mathrm{K})$. For review, see $[2,3]$.

ted by a laser so that they stop near the centre of the trap. The current is then suddenly applied. This results in the confinement of $\cong 10000$ atoms with a temperature of $\cong 17 \mathrm{mK}$ for a duration of $\cong$ $1 \mathrm{~s}$. Trapping time was limited by collisions with the residual background gas (pressure $\cong 10^{-8}$ Torr).

Another sodium magnetic trap has been built at M.I.T. in D. Pritchard's group [6]. It uses a superconducting magnet $\left(\triangle B \cong 1300\right.$ Gauss, $\left.U_{M}=0.1 \mathrm{~K}\right)$ and the trapped atoms are permanently cooled by a laser. It confines $10^{9}$ atoms for several minutes, at a temperature of $3 \mathrm{mK}$. This temperature could even be reduced in the future with the use of a new cooling method suggested by Pritchard [7] which includes both a laser and a microwave excitation.

Finally, an atomic hydrogen magnetic trap has recently been realized in $D$. Kleppner's group, also at M.I.T. [8]. Since no $\mathrm{CW}$ laser radiation is yet available at the Lyman $\alpha$ line, all the cooling pro- cesses have to be done with the usual cryogenic methods. Hydrogen atoms are produced in the trap by a pulsed discharge in a cell containing molecular hydrogen and helium, cooled by a dilution refrigerator below $0.2 \mathrm{~K}$. The trap has a depth of $0.58 \mathrm{~K}$, and it has been possible to contain $5 \times 10^{12}$ atoms for 20 minutes. Furthermore, evaporative cooling lowers the temperature of these trapped atoms to $40 \mathrm{mK}$. The cooling results from the very simple fact that, when a collision between initially trapped atoms produces a high velocity atom, this atom leaves the trap, carrying more energy than possessed by an average velocity atom in the trap. The density of trapped atoms $\left(10^{13} \mathrm{at} / \mathrm{cm}^{3}\right)$ remains for the moment far below the Bose Einstein condensation limit at this temperature $\left(5 \times 10^{17} \mathrm{at} / \mathrm{cm}^{3}\right)$, but further evaporative cooling should lead to much lower temperature in the near future, so that quantum effects should become observable.

\section{Optical Traps}

Like magnetic traps, optical confinement of neutral atoms usually relies on the curving of the atomic energy levels. For stable trapping, a potential with a local minimum at the centre of the trap is necessary. Intense laser beams provide such a potential, which can be written near a resonant transition [2]:

$$
U_{L}(\vec{r})=(\hbar \Delta / 2) \log (1+s(\vec{r}))
$$

where $\Delta=\omega_{\mathrm{L}}-\omega_{\mathrm{A}}$ is the detuning between the laser $\left(\omega_{L}\right)$ and atomic $\left(\omega_{A}\right)$ frequencies, and $s$ is the saturation parameter:

$$
s(\vec{r})=\omega_{1}^{2}(\vec{r}) / 2\left(\Delta^{2}+\Gamma^{2} / 4\right)
$$

In eq. $2, \omega_{1}(\vec{r})$ is the local Rabi frequency at point $r$, proportional to the atomic dipole $\vec{d}$ and to the laser electric field

$\vec{E}[\vec{r})\left(\omega_{1}(\vec{r})=\vec{d} \cdot \vec{E}(\vec{r}) / \hbar\right)$;

$\Gamma^{-1}$ is the spontaneous lifetime of the excited state of the atomic transition.

The force $\nabla U_{L}$ deriving from the potential $U_{\mathrm{L}}(\vec{r})$, is the so-called dipole force. Depending on the sign of the detuning, this force attracts the atoms towards the high intensity regions (for $\Delta$ $<0$ ), or repels the atoms from these regions (for $\Delta>0$ ). If we take a strongly focussed laser beam with a negative detuning (Fig. 2), the central point in the waist plane is a local intensity maximum and thus a stable trapping point. This is the principle of the first optical atomic trap, which was recently demonstrated by a group at Bell Laboratories [9]. In this experiment, about 500 sodium atoms were trapped in a volume of $1000 \mu \mathrm{m}^{3}$ for a few seconds. The $220 \mathrm{~mW}$ dye laser is focussed to a $10 \mu \mathrm{m}$ waist radius, and it is tuned to a frequency of $650 \mathrm{GHz}$ below the sodium $\mathrm{D}_{2}$ line. This leads to an optical well depth of $5 \mathrm{mK}$,

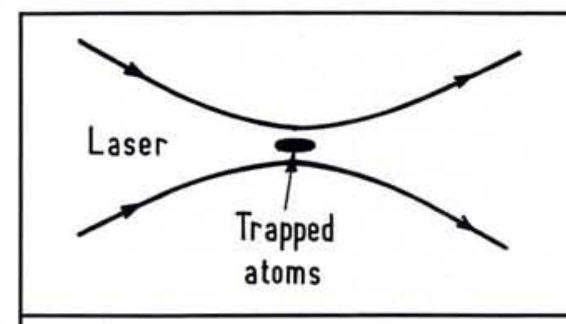

Fig. 2 - The first realized laser trap [9] confines the atoms in the high intensity region located near the focal plane of a strongly focussed laser beam. In this experiment, the detuning of the laser $\omega_{L}-\omega_{A}$ was negative, and sufficiently large so that one could neglect the scattering force (see Fig. 1) compared to the dipole force creating the trapping potential. Additional laser cooling was also applied, to compensate for the heating due to the fluctuations of radiative forces. 


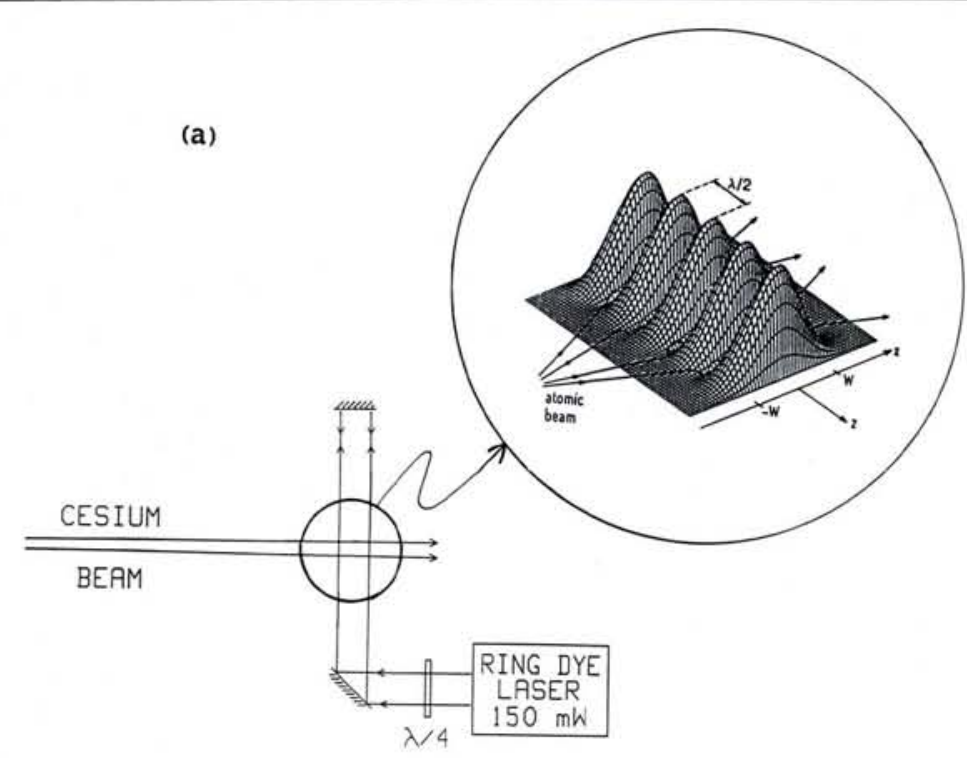

(b)

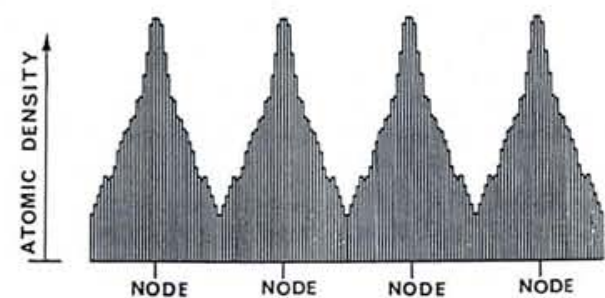

Fig. $3-A$ strong laser standing wave detuned blue from resonance $\left(\omega_{L}>\omega_{A}\right)$ creates a periodic potential with minima at the nodes and maxima at the antinodes. In the experiment realized in Paris [10], a well collimated atomic beam is crossed at right angles by this strong standing wave (Fig. 3(a)). For a low enough transverse kinetic energy, atoms get channelled near the nodes of the standing wave. Using a position imaging technique based on light-shifts, it is possible to reconstruct the atomic spatial distribution (Fig. 3 (b)). It is strongly peaked around the nodes with a half width-half maximum $\Delta x / \lambda=1 / 10$. For a red detuning $\left(\omega_{\mathrm{L}}<\omega_{\mathrm{A}}\right)$, a similar channelling around the antinodes has also been observed.

and a corresponding maximum trappable velocity of about $2.7 \mathrm{~m} / \mathrm{s}$. Here again the loading of the trap is made possible by a preliminary laser cooling of the sodium atoms.

Laser cooling of the trapped atoms is permanently achieved by "optical molasses" (see Fig. 1b). Such a cooling, which is optional for magnetic traps, is indispensable for laser traps. This arises from the nature of the optical trap: the potential (1) is a time averaged potential, and the corresponding dipole force fluctuates around its average value. These fluctuations originate from the random character of the spontaneous emission processes inherent to laser-atom interactions. They produce some heating which, if not compensated for, would make the atom quickly leak out of the shallow trap. For this reason, the trapp- ing beam is periodically turned on and off so that the atom sees half of the time a restoring force and half of the time a cooling force. If the on-time is short compared to the period of oscillation of the atoms in the trapping potential, the atom has a damped oscillatory motion, in a well which is simply half the $\mathrm{CW}$ potential well given by eq. 1 .

The volume of this optical trap, although small, is still four orders of magnitude larger than the $\lambda^{3}$ volume, where $\lambda$ is the wavelength of the trapping laser. This trap does not therefore answer the old and intringuing question: is it possible to trap atoms with light on the optical wavelength scale? The answer is not yet known, but a group led by C. Cohen-Tannoudji in Paris has realized a one-dimension trap of this kind [10]. It uses again the potential (1) provided now by an intense laser standing wave. This potential is then periodic and, for positive detunings, it varies from its minimal value (at nodes) to its maximal value (at antinodes) over a distance $\lambda / 2$. In the Paris experiment, a caesium atomic beam is crossed at right angles by this intense laser standing wave (see Fig. 3). If the transverse kinetic energy of the atoms is smaller than the potential height, atoms get channelled near the nodes of the standing wave. The channelling has been observed using a very sensitive spectroscopic technique based on the atomic level light shifts induced by the intense standing wave. The atomic spatial distribution appears to be strongly peaked around the nodes, with a half width at half maximum that is $\Delta x=80 \mathrm{~nm}(\Delta x / \lambda \cong 1 / 10)$ (Fig. 3b). The extension to three dimensions of this technique using laser decelerated and laser cooled atoms is now being investigated in Paris.

Up to now, we have only been considering light traps based on the potential (1) arising from the dipole force. This force can be shown to result from the inphase component of the laser-induced atomic dipole moment. Another kind of light trap makes use of the "scattering force", proportional to the quadrature component of the induced dipole and to the local Poynting vector of the laser wave. The scattering force is the force used for atomic beam deceleration or optical molasses, as shown in Fig. 1. The history of traps based on the scattering force is rather amusing since it illustrates very well the non-straight-forward evolution of the physical concepts in this research area. Up to 1983 , several propositions for a laser trap using only this scattering force had been made, but in 1983, Ashkin and Gordon showed that they would all leak, owing to an optical equivalent of the electrostatic Earnshaw theorem [11]: for a static laser beam, the divergence of the associated Poynting vector $\vec{\pi}$ is zero. As a consequence, if the scattering force acting on the atom is strictly proportionnal to $\vec{\pi}$, as is usually assumed, this force also has a null divergence. Applied to the centre of the trap, this relation precludes a restoring force operating in all directions, and therefore stable trapping.

Fortunately, many schemes were then proposed to circumvent the Optical Earnshaw Theorem. They use for instance time dependent laser intensity, in analogy with radio frequency traps for ions, or optical pumping mechanisms preventing a strict proportionality between the force and the Poynting vector. As an example, Fig. 4 shows a scheme 


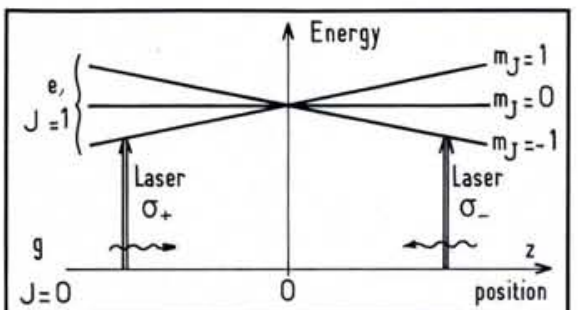

Fig. 4 - One-dimensional principle of a magneto-optic trap using a (ground state, $J=0$ ) $\rightarrow$ (excited state, $J=1$ ) transition, in a magnetic field gradient. The magnetic field is zero at the centre of the trap and varies linearly along the trap. Atoms are irradiated by two counter-propagating laser beams, detuned red from resonance $\left(\omega_{\mathrm{L}}<\omega_{\mathrm{A}}\right)$ and respectively polarized $\sigma_{+}$and $\sigma_{-}$along the $z$-axis. Due to this choice of polarizations, atoms on the left (resp-right) of the trap are resonant with the laser coming from the left (resp-right) and are thus pushed towards the centre.

which associates the scattering force and the Zeeman effect. This "magnetooptic" trap has recently been demonstrated in a collaboration between Bell Laboratories and M.IT. [12]. The trap volume, much bigger than for a dipole force trap, can be as large as $30 \mathrm{~mm}^{3}$. The trap contains up to $2 \times 10^{6}$ atoms, which leads to densities at the centre of about $2 \times 10^{11}$ atoms $/ \mathrm{cm}^{3}$. The estimated temperature for the trapped atoms is about $1 \mathrm{mK}$.

To summarize, laser traps can now confine neutral atoms for minutes with a temperature around or below the milliKelvin range. So far, densities of up to $n$ $\cong 10^{11}-10^{12}$ atoms $/ \mathrm{cm}^{3}$ have been obtained in both magnetic and optical traps. As already mentioned the very exciting goal of Bose Einstein condensation is still about four to five orders of magnitude ahead for the $n / T^{3 / 2}$ ratio. In order to reach it, a further increase of density and/or decrease of temperature has still to be realized. Let us briefly outline these two possibilities:

- Is it possible to increase the density?

Some authors think that recombination processes, such as the formation of molecules $\left(\mathrm{H}_{2}, \mathrm{Na}_{2}\right)$ or associative ionization will appear soon. These recombination products would not be trapped. This will therefore be a leakage process which will limit the atomic density. In any case, experiments with trapped cold-atoms open the way to collision studies in a very new regime ( $\mathrm{T} \leqq 1 \mathrm{mK}$ ). At these very small energies, the collisions will be sensitive to the long range interaction potentials which are not well known for the moment.

\section{- Is it possible to cool further?}

Laser cooling has proved to be very efficient in most of these trapping experiments and numerous proposals have been made to enter the nanoKelvin microKelvin regime. An obvious way is to use longer lived excited states since the radiative cooling limiting temperature is proportional to the upper state natural line width. Also much less energetic photons like radio frequency photons may be used. Most of the proposed methods will take advantage of the long storage time now allowed by these traps. Achieving temperatures in the microKelvin range will benefit not only the Bose-Einstein condensation "quest". At such temperatures, the atomic De Broglie wavelength becomes very large, typically of the order of optical wavelengths. One can think of doing interferometry with these atoms in a similar way that cold neutrons have been shown to interfere. With such a large De Broglie wavelength, it is also conceivable that cold atoms might bounce elastically on solid surfaces since microscopic surface defects would then be too small to be "seen" by the colliding atom. If this is true, one could imagine storing these cold atoms in a box!

In a different domain, spectroscopists and atomic clock physicists hope to improve the precision of their experiments with laser cooled neutral atoms or ions. With cold particles, the better control of the second order Doppler effect (relativistic time dilation) and the long interaction time should allow a resolution of $10^{14}-10^{15}$ for atomic lines in the visible range. In addition, neutral atoms can be confined to high densities unlike ions for which the density is limited by space charge effects. Using such narrow atomic transitions, one hopes to lock lasers with a relative stability in the range $\Delta v / v$ $\cong 10^{-16}-10^{-17}$. These clocks would in turn enable some very fundamental tests in physics.

In conclusion, it appears that the physics involved in the trapping of neutral atoms is now rather well understood. Both magnetic and light traps work in a predictable and reproducible way. On the other hand, these recent techniques open several new fields in domains as different as statistical physics, molecular physics, atomic interferometry and metrology. Judging from the two dozens of laboratories working now in the field of neutral atom cooling and trapping, we

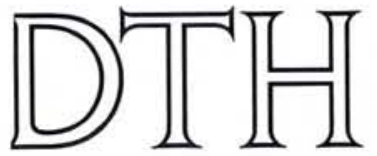

Technical University of Denmark

\section{Associate Professor in Surface Physics}

At the Technical University of Denmark a new Surface Science Research Group is being created. When fully staffed it is planned to consist of 3-4 faculty positions. The second of these, at the associate professor (lecturer) level, is now open for applications at the Laboratory of Applied Physics.

Applicants are expected to have several years of post doctoral research experience within Surface Physics. The successful applicant is required to have experience within dynamic processes at solid surfaces, especially adsorption- desorption- and reaction dynamics, chemisorption, molecular beam scattering and surface lattice dynamics.

The applicant must be able to undertake teaching in elementary and advanced physics courses.

Curriculum vitae including relevant information (publication list and publications), and an account of teaching experiences all in three copies is to be directed to:

Faculty of Basic Sciences - Technical University of Denmark Building 101 - DK-2800 Lyngby - Denmark

no later than September 1, 1988.

Further information can be obtained from

Professor Jens Nørskov or Dr. lb Chorkendorff,

Laboratory of Applied Physics, Building 307.

Technical University of Denmark, DK-2800 Lyngby, Denmark Tel. : + (45) (2) 882488 ext. 2301 or 2323 . 
expect exciting new developments in the near future.

\section{REFERENCES}

[1] Phillips W.D., Prodan J.V. and Metcalf H.J., J. Opt. Soc. Am. B. 2 (1985) 1751.

[2] Stenholm S., Rev. Mod. Phys. 58 (1986) 699.

[3] Wineland D.J. and Itano W.M., Phys. Today, June 1987, p. 34.

[4] Kügler K.J., Paul W. and Trinks U., Phys. Lett. 72B (1978) 422.

[5] Prodan J., Migdall A., Phillips W.D., So I., Metcalf H. and Dalibard J., Phys. Rev. Lett. 54 (1985) 992.

[6] Bagnato V.S., Lafyatis G.P., Martin A.G., Raab E.L., Ahamd-Bitar R.N. and Pritchard D.E., Phys. Rev. Lett. 58 (1987) 2194.

[7] Pritchard D.E., Phys. Rev. Lett. 51 (1983) 1336.

[8] Hess H.F., Kochanski G.P., Doyle J.M., Masuhara N., Kleppner D. and Greytak T.J., Phys. Rev. Lett. 59 (1987) 672.

[9] Chu S., Bjorkholm J.E., Ashkin A. and Cable A., Phys. Rev. Lett. 57 (1986) 314.

[10] Salomon C., Dalibard J., Aspect A., Metcalf $\mathrm{H}$. and Cohen-Tannoudji C., Phys. Rev. Lett. 59 (1987) 1659.

[11] Ashkin A. and Gordon J.P., Opt. Lett. 8 (1983) 511.

[12] Raab E.L., Prentiss M., Cable A., Chu S. and Pritchard D.E. Phys. Rev. Lett. 59 (1987) 2631.

\section{Chemical Physics}

The Board of the Chemical Physics Section of the Atomic and Molecular Physics Division have decided to coopt the following:

E.A.G. Armour, University, Nottingham

E. Kochanki, University, Strasbourg

A. Varandas, University, Coimbra

A General Assembly of the Chemical Physics Section will be held during the ECAMP III Conference that takes place in the first week of April 1989 in Bordeaux. The exact details will be announced there. A Symposium on Applied Theoretical Chemistry is also being organized as a series of parallel sessions to ECAMP III.

EPS Divisions, Sections and Group

Astronomy and Astrophysics Division Solar Physics Section

Solar Physics Section
Atomic and Molecular Physics Division

Atomic and Molecular Physics Divisi

Clectronic and Atomic Collisions

Molecular Physics

Computational Physics Group

Condensed Matter Division

Liquids Section

Low Temperature Physics Section

Macromolecular Physics

Magnetism

Metal Physics

Semiconductors and insulators

Surfaces and Interfaces

High Energy \& Particle Physics Division

Interdiv. Group on Exptl. Phys. Control Systems

Interdiv. Group on Physics for Development

Nuclear Physics Division

Optics Division

Optics Division

Quantum Electronics Division

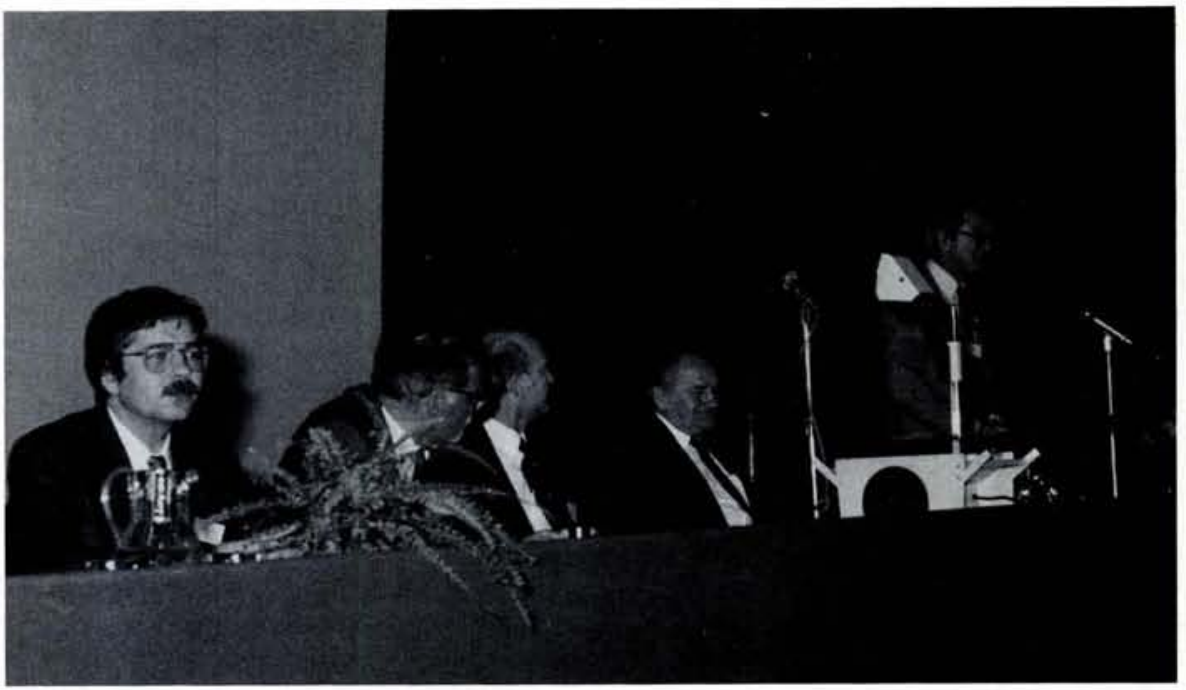

Presentation of the 1988 Hewlett-Packard Europhysics Prize during the 8th EPS General Condensed Matter Physics Conference held this year from 6-9 April in Budapest. At the microphone A.R. Mackintosh, Chairman of the Selection Committee and to his right: W. Buckel, retiring President of EPS, N. Kroo, Chairman of the Conference, and the two award winners, K. Alex Müller and J. Georg Bednorz.

\section{Europhysics News welcomes Readers' letters}

\section{Quantum Electronics}

As a result of the elections to the Board of the Quantum Electronics Division, the new members are:

J.S. Bakos, Central Research Institute for

Physics, Budapest

D.R. Hall, Heriot-Watt University, Edinburgh

P. Knight, Imperial College, London

F.K. Reinhart, EPFL, Lausanne

J.P. Woerdman, University, Leiden

A full list of the Board will appear in the Directory that will be published in the June issue of Europhysics News.

The Chairman, $\mathrm{H}$. Walther and the Secretary, F.R. Aussenegg announce that the Division will be holding a Business Session on Tuesday, 13 September 1988 in conjunction with EQEC ' 88 at Hannover. Members please note.

Europhysics News is the official journal of the European Physical Society which comprises 29 National Societies, Academies and Group, about 4000 Individual Members and 75 Associate Members. Governing bodies of EPS are the General Meeting, Council and an elected Executive Committee responsible for detailed policy. EPS promotes the collaboration of physicists throughout Europe, organising and harmonising conferences and publications, improving physics education, rences and encouraging physics applications, awarding scholarships to sponsored schools in Erice. EPS publishes in addition to Europhys. News, Europhysics Letters (in partnership with national societies), European Journal of Physics (in partnership with The UK Inst. of Phys.) and European Conference Abstracts. Individual Memand Europan Conference Abstracts. Individual Meminsts.: Sw.Fr. 90.-la), Europhys. Lett. at Sw.Fr. 125.-1a (insts. 1050.-), rebates on many other publications and on conference fees. Annual EPS membership fee for Individual Members belonging to an EPS member society is: Sw.Fr. 44-; independent members: Sw.Fr. 132 members of a Collaborating Society: Sw.Fr. 55.-

\section{Contents}

From the President

EPS Council Meets in Dresden

The Physics of Ice

61

H-P Prize: Call for Nominations 64

The Hydrogen-Metal

Interaction

Meetings Up-Date \& Corrections

Trapping Neutral Atoms with Electromagnetic Fields

H-P Prize 1988 Presentation

Divisional Elections and Meetings

Editor: E.N. Shaw

Editorial Board:

A. Baratoff, F. James, M. Lehmann,

M. Mayor. J. Muller, M. Siegrist Secretariat

Address: EUROPEAN PHYSICAL SOCIETY P.O. Box 69 , CH-1213 Petit-Lancy 2

Switzerland

Telephone: Geneva (22) 931130

Telex: 428024 eps ch

Telefax: (22) 931317

Printed by: Pfirter frères sa

$\mathrm{CH}-1213$ Petit-Lancy/Switzerland
Editorial and Advertising Office at the EPS 